\title{
A NEW CANONICAL FORM OF THE ELLIPTIC INTEGRAL*
}

\author{
BESSIE I. MILLER
}

\section{INTRODUCTION}

The elliptic norm curve $Q_{n}$ in space $S_{n-1}$ admits a group $G_{2 n^{2}}$ of collineations, and in fact there is a single infinity of such curves which admit the same group. A particular $Q_{n}$ of the family is distinguished by a value of the parameter $\tau$, itself an elliptic modular function defined by the modular group congruent to identity $(\bmod . n)$.

In the group $G_{2 n^{2}}$, there are certain involutory collineations with two fixed spaces. If $Q_{n}$ is projected from one fixed space upon the other, a family of rational curves $R_{m}$ mapping the family of $Q_{n}$ 's is obtained. The quadratic irrationality separating involutory pairs on $Q_{n}$ involves the modulus $\tau$, and the parameter $t$ of the $R_{m}$. When the genus of the modular group is zero and $n=3,4,5$, the irrationality can be used to define the elliptic parameter

$$
u=\int \frac{(t d t)}{\sqrt{(t \tau) \alpha_{\tau}^{r-3} \alpha_{t}^{3}}},
$$

where $\alpha_{t}^{r}$ is the tetrahedral, octahedral, or icosahedral form. This is in contrast with Klein's form as developed by Bianchi, for there the normal elliptic integral is a rational curvilinear integral taken along an elliptic curve.

A comparison of the two integrals is more illuminating if carried out for a special case. Let $Q_{n}$ be $Q_{5}$ in $S_{4}$. Let $\phi_{i}\left(x_{j}, a\right)=0,(i, j=0, \cdots 4)$ be five quadrics intersecting in $Q_{5}$, where according to Bianchi's notation $x_{j}$ are the variables, $a$ the modulus. After a suitable transformation of coördinates the icosahedral form which appears in the irrationality is

$$
\alpha_{t}^{12}=t_{1} t_{2}\left(t_{1}^{10}+11 t_{1}^{5} t_{2}^{5}-t_{2}^{10}\right) .
$$

The integral $u$ involving $\tau \equiv a$ explicitly in a rather simple form is uniquely defined. Moreover it is invariant under all cogredient transformations of $t$ and $\tau$, which leave $\alpha_{x}^{12}$ unaltered, i. e., the sixty transformations of the icosahedral group applied simultaneously to $t$ and $\tau$, the parameter of the doubly covered conic $R_{2}$ and the modulus of $Q_{5}$ respectively, leave $u$ unaltered.

Consider now Bianchi's integral, defined as

\footnotetext{
* Presented to the Society, December 27, 1915.
} 


$$
U=C \int \frac{(w d v-v d w)}{\left(\phi_{0} \phi_{1} \phi_{2} w v\right)}
$$

where $C$ is a constant, $w$ and $v$ any two expressions linear in $x_{j}$, and the denominator is the functional determinant of $\phi_{0}, \phi_{1}, \phi_{2}, w, v$. Different expressions for $U$ can be obtained by making different choices for $w$ and $v$. Hence there is no unique form for $U$. Under the $G_{50}$ of collineations on $x_{j}$ and under the transformations of $a \equiv \tau, U$ assumes various conjugate forms.

Hence $u$ has the advantage over $U$ in simplicity and uniqueness of form, and also in its invariancy under transformations.

In section I by a study of the integral $u$ some interesting results are obtained. The modular equation connecting $\tau$ and $J$, the absolute invariant of $u$, can be deduced as the result of the binary syzygy of lowest weight connecting the concomitants of $\alpha_{x}^{r}$. The requirement that the Riemann surface attached to the modular equation be regular leads to the modular equations associated with the regular bodies. It is then possible to eliminate the more tedious individual proofs used by Bianchi in the discussion of the moduli of $Q_{3}$ and $Q_{5}$ to show that these moduli are the tetrahedral and icosahedral irrationalities respectively. In fact the algebraic discussion carried out once for $\alpha_{x}^{r}$ is complete for factor groups of genus zero, which have been discussed by Klein, i. e., those isomorphic with the groups associated with the regular bodies, namely the one dihedral group $G_{6}$, and the tetrahedral, octahedral, and icosahedral groups.

In sections II, III, IV, it is shown that the canonical form $u$ occurs naturally in connection with the elliptic norm curves $Q_{3}, Q_{4}, Q_{5}$ and in section III the possibility of extending the canonical form to larger values of the genus is indicated.

Whenever possible the notation conforms to that of Klein-Fricke, Bianchi, and Grace and Young.*

\section{A SYZYGY}

1. Consider the elliptic integral of the first kind in the form given in the introduction. $\dagger$ The form under the radical sign, since it is a quartic in $t$,

* Abbreviations and references:

K. F. Vorlesungen über die Theorie der elliptischen Modulfunctionen, by Klein and Fricke.

B. Über die Modulformen dritter und fünfter Stufe des elliptischen Integrals erster Gattung, by Bianchi in Mathematische Annalen, vol. 17 (1880), pp. 234262.

G. \& Y. Algebra of Invariants, by Grace and Young.

$\dagger$ The integral $u$ can be reduced to Weierstrass's canonical form by means of the transformation $x_{1}=\alpha_{\tau}^{n-1} \alpha_{t}, x_{2}=(t \tau)$. (Vide Clebsch, Theorie der binären algebraischen Formen, §81-83, for similar transformations.) 
has two invariants* $g_{2}$ and $g_{3}$, the former of degree $2(n-2)$ in $\tau$, the latter of degree $3(n-2)$ in $\tau$. But $f \equiv \alpha_{\tau}^{n}$ has one covariant, $H$, of degree $2(n-2)$ in $\tau$, and one covariant, $(f, H)^{1}$, of degree $3(n-2)$ in $\tau$. Hence $g_{2}$ must be some multiple of $H ; g_{3}$ some multiple of $(f, H)^{1}$. The discriminant, $\Delta$, of a quartic can be expressed in terms of $g_{2}^{3}$ and $g_{3}^{2}$. The absolute invariant $J$ is then defined by the syzygy

$$
J: J-1: 1:: g_{2}^{3}: 27 g_{3}^{2}: \Delta .
$$

In order to obtain an analogous syzygy involving the transvectants of $f$, it is necessary to obtain the expressions for $g_{2}, g_{3}$, and $\Delta$, in terms of the transvectants of $f$ and to determine some relation between these forms.

2. Let

$$
\begin{aligned}
f & \equiv \alpha_{\tau}^{n} \equiv \beta_{\tau}^{n} \equiv \gamma_{\tau}^{n} \equiv \text { etc., } \\
g & \equiv \alpha_{\tau}^{n-3} \alpha_{t}^{3}(t \tau) \equiv \beta_{\tau}^{n-3} \beta_{t}^{3}(t \tau) \equiv \text { etc. }, \\
H & \equiv(\alpha \beta)^{2} \alpha_{\tau}^{n-2} \beta_{\tau}^{n-2}=(f, f)^{2}, \\
H_{g} & \equiv(g, g)^{2}, \\
\tilde{t} & \equiv(\alpha \beta)^{2}(\gamma \alpha) \alpha_{\tau}^{n-3} \beta_{\tau}^{n-2} \gamma_{\tau}^{n-1}=(f, H)^{1}, \\
i & \equiv(\alpha \beta)^{4} \alpha_{\tau}^{n-4} \beta_{\tau}^{n-4}=(f, f)^{4}, \\
j & \equiv(\alpha \beta)^{2}(\beta \gamma)^{2}(\gamma \alpha)^{2} \alpha_{\tau}^{n-4} \beta_{\tau}^{n-4} \gamma_{\tau}^{n-4}=(f, i)^{2}, \\
k & \equiv(\alpha \beta)^{6} \alpha_{\tau}^{n-6} \beta_{\tau}^{n-6}=(f, f)^{6} .
\end{aligned}
$$

According to Klein $(g, g)^{4}$ equals $2 g_{2}$ and $\left(H_{g}, g\right)^{4}$ equals $4 g_{3}$.

$$
\begin{gathered}
(g, g)^{4}=\alpha_{\tau}^{n-3} \beta_{\tau}^{n-3}\left(\alpha_{\tau}^{3}(t \tau), \beta_{\tau}^{3}(t \tau)\right)^{1}=-\frac{18}{4 !}(\alpha \beta)^{2} \alpha_{\tau}^{n-2} \beta_{\tau}^{n-2} . \\
\therefore g_{2}=-\frac{3}{8} H . \\
H_{\sigma} \dagger=\frac{1}{2}(\alpha \beta)^{2} \alpha_{\tau}^{n-3} \beta_{\tau}^{n-3} \alpha_{t} \beta_{t}(t \tau)^{2}, \\
\left(H_{g}, g\right)^{4}=\frac{6}{4 !}(\alpha \beta)^{2}(\gamma \alpha) \alpha_{\tau}^{n-3} \beta_{\tau}^{n-2} \gamma_{\tau}^{n-1} . \\
\therefore g_{3}=\frac{1}{16}(f, H)^{1}=\frac{1}{16} \tilde{t} .
\end{gathered}
$$

Now $\tilde{t}$ is a jacobian. Therefore its square $\ddagger$ is reducible. In fact

$$
-2 \tilde{t^{2}}-H^{3}=(H, H)^{2} f^{2}-2(f, H)^{2} f H .
$$

Let $\Delta^{\prime}=2 \tilde{t^{2}}+H^{3}$ and $J=H^{3} / \Delta^{\prime}$. Then $J-1=-2 \tilde{t^{2}} / \Delta^{\prime}$ and the following proportion can be written

* K. F., I, pp. 13-15.

$\dagger$ G. \& Y., $\$ \& 43,49$, for methods of calculating transvectants.

† G. \& Y., \& 78. 


$$
J: J-1: 1=H^{3}:-2 \tilde{t^{2}}: \Delta^{\prime} .
$$

This is assuredly a relation between the hessian and jacobian of an nic, similar to the one existing between the invariants, $g_{2}$ and $g_{3}$, of a quartic. Corresponding to the discriminant $\Delta$ of the quartic is the form $\Delta^{\prime}$, expressed by (1) in terms of transvectants which are reducible. Hence before (2) is discussed, the reduction of $(H, H)^{2}$ and of $(f, H)^{2}$ is made.

3. The reduction just mentioned can be outlined as follows:

$$
\begin{aligned}
(f, H)^{2} & =\frac{1}{2 n-5}(\alpha \beta)^{2}\left\{(n-3)(\alpha \gamma)^{2} \alpha_{\tau}^{n-4} \beta_{\tau}^{n-2} \gamma_{\tau}^{n-2}\right. \\
& \left.+(n-2)(\alpha \gamma)(\beta \gamma) \alpha_{\tau}^{n-3} \beta_{\tau}^{n-3} \gamma_{\tau}^{n-2}\right\}^{*} \\
& =(\alpha \beta)^{2}(\alpha \gamma)^{2} \alpha_{\tau}^{n-4} \beta_{\tau}^{n-2} \gamma_{\tau}^{n-2}-\frac{n-2}{2(2 n-5)} \text { if } \\
& =(\alpha \beta)^{2}(\alpha \gamma)^{2} \beta_{\tau}^{2} \gamma_{\tau}^{2} \alpha_{\tau}^{n-4} \beta_{\tau}^{n-4} \gamma_{\tau}^{n-4}-\frac{n-2}{2(2 n-5)} \text { if } \dagger \\
& =\frac{1}{2} i f-2(\beta \gamma)^{2}(\alpha \beta)(\alpha \gamma) \alpha_{\tau}^{n-2} \beta_{\tau}^{n-3} \gamma_{\tau}^{n-3}-\frac{n-2}{2(2 n-5)} \text { if } \ddagger \\
& =\frac{n-3}{2(2 n-5)} \text { if. }
\end{aligned}
$$

The reduction of $(H, H)^{2}$ is accomplished by means of Gordan's series. $\S$ Since $(H, H)^{2}=\left((f, f)^{2}, H\right)^{2}$, the first series used is

This gives

$$
\left(\begin{array}{ccc}
f & f & H \\
n & n & 2 n-4 \\
0 & 2 & 2
\end{array}\right) .
$$

$$
\begin{aligned}
(H, H)^{2}+\frac{n-2}{2(2 n-5)} i H=\left((f, H)^{2}, f\right)^{2} & +\frac{4(n-3)}{3 n-8}\left((f, H)^{3}, f\right)^{1} \\
& +\frac{2(2 n-7)}{3(3 n-10)}\left((f, H)^{4}, f\right)^{0} .
\end{aligned}
$$

The terms on the right-hand side of (3) must be reduced in turn so that (3) may contain only the transvectants enumerated in $\S 2$. It is found that

* G. \& Y., $\$ 22$. Apply identity III.

† Square identity III (loc. cit.) and apply it.

$\ddagger$ G. \& Y., \& 22. Rewrite identity I thus: $(\beta \gamma) \alpha_{\tau}=(\beta \alpha) \gamma_{\tau}-(\gamma \alpha) \beta_{\tau}$ and multiply by $(\beta \gamma)(\alpha \beta)(\alpha \gamma)$ before applying.

§ G. \& Y., § 54 . 


$$
\begin{aligned}
\left((f, H)^{2}, f\right)^{2}=\frac{1}{2(2 n-5)(3 n-8)}\{n(n-3) i H \\
\left.-\frac{(2 n-8)(2 n-9)}{3} j f+\frac{(n-4)(n-5)}{3} k f^{2}\right\} .
\end{aligned}
$$

The transvectant $(f, H)^{3}$ is given by the series

and equals

$$
\left(\begin{array}{lll}
f & f & f \\
n & n & n \\
0 & 2 & 3
\end{array}\right),
$$

$$
\begin{gathered}
\frac{n-4}{2(2 n-5)}(f, i)^{1}, \\
\left((f, H)^{3}, f\right)^{1}=\left(\frac{n-4}{2(2 n-5)}(f, i)^{1}, f\right)^{1 *} \\
=\frac{n-4}{2(2 n-5)}\left\{-\frac{2 n-9}{3 n-10}\left[-j+\frac{n-5}{2(2 n-9)} k f\right]+\frac{1}{2} i H\right\} .
\end{gathered}
$$

The transvectant $(f, H)^{4}$ is given by the series

$$
\begin{gathered}
\left(\begin{array}{ccc}
f & f & f \\
n & n & n \\
0 & 4 & 2
\end{array}\right) . \\
\therefore(f, H)^{4}=-\frac{n-1}{2 n-5}(i, f)^{2}+\frac{(3 n-10)(n-5)}{4(2 n-7)(2 n-9)} k f \\
\quad=\frac{n-1}{2 n-5} j+\frac{(n-4)(n-5)}{4(2 n-7)(2 n-9)} k f .
\end{gathered}
$$

If the values of the different transvectants are substituted in (3), the following result is obtained:

$$
(H, H)^{2}=-\frac{1}{2(2 n-5)} i H+\frac{1}{3} j f .
$$

It is interesting to notice that the term $k f^{2}$ has the coefficient zero. Therefore $k$, a form which does not exist in the case of the quartic, does not appear in the final result.

4. The relation between $t$ and $H$ is given by (1). If now the results of $\$ \S 2$ and 3 are used, (1) can be replaced by

$$
\tilde{2 t^{3}}+H^{2}=f^{2}\left(\frac{1}{2} i H-\frac{1}{3} j f\right) \text {. }
$$

Then (2) can be written as follows:

* See G. \& Y., $\$ 77$ (XIX), for the formula used to obtain the result given above. 


$$
J: J-1: 1=6 H^{3}:-1 \tilde{2 \tilde{t}^{2}}: f^{2}(3 i H-2 j f) .
$$

If the equivalents of $H$ and $\tilde{t}$ (vide $\S 2$ ) are introduced in (4), then the usual form of the syzygy existing between the invariants of the quartic is obtained. Thus (4) is the desired syzygy between the hessian and jacobian of an nic. It shows that, as $H$ and $\tilde{t}$ correspond to $g_{2}$ and $g_{3}$ respectively, $\Delta^{\prime}$ corresponds to $\Delta$.

5. The syzygy (4) containing the parameter $\tau$ and the invariant $J$ is for a given $J$ an algebraic equation of degree $6(n-2)$, with roots which are algebraic functions of $J$. It can be mapped on a $6(n-2)$-sheeted Riemann surface. The $k$-fold roots of (4) and the values of $J$ giving the branch points of the surface must be determined.

Since a $k$-fold root of $(4)$ is a $(k-1)$-fold root of the transvectant $\left(H^{3}, \tilde{t^{2}}\right)^{1}$, the reduction of this transvectant is necessary. Let

Then

$$
\begin{aligned}
H & \equiv(\alpha \beta)^{2} \alpha_{\tau}^{n-2} \beta_{\tau}^{n-2} \equiv h_{\tau}^{2(n-2)} \\
\tilde{t} & \equiv(\delta \epsilon)^{2}(\eta \delta) \delta_{\tau}^{n-3} \epsilon_{\tau}^{n-2} \eta_{\tau}^{n-1} \equiv t_{\tau}^{3(n-2)}
\end{aligned}
$$

$$
\begin{aligned}
\left(H^{3}, \tilde{t}^{2}\right)^{1}= & \frac{H^{2} \tilde{t}(\alpha \beta)^{2}(\delta \epsilon)^{2}(\eta \delta)}{6(n-2)} \alpha_{\tau}^{n-3} \beta_{\tau}^{n-3}\left\{(n-3)(\alpha \delta) \beta_{\tau} \delta_{\tau}^{n-4} \epsilon_{\tau}^{n-2} \eta_{\tau}^{n-1}\right. \\
& +(n-2)(\alpha \epsilon) \beta_{\tau} \delta_{\tau}^{n-3} \epsilon_{\tau}^{n-3} \eta_{\tau}^{n-1}+(n-1)(\alpha \eta) \beta_{\tau} \delta_{\tau}^{n-3} \epsilon_{\tau}^{n-2} \eta_{\tau}^{n-2} \\
& +(n-3)(\beta \delta) \alpha_{\tau} \delta_{\tau}^{n-4} \epsilon_{\tau}^{n-2} \eta_{\tau}^{n-1}+(n-2)(\beta \epsilon) \alpha_{\tau} \delta_{\tau}^{n-3} \epsilon_{\tau}^{n-3} \eta_{\tau}^{n-1} \\
& \left.+(n-1)(\beta \eta) \alpha_{\tau} \delta_{\tau}^{n-3} \epsilon_{\tau}^{n-2} \eta_{\tau}^{n-2}\right\}=H^{2} \tilde{t}(H, \tilde{t})^{1} \\
= & H^{2} \tilde{t f}\left\{\frac{n-3}{3(n-2)} j f-\frac{2 n-3}{3(n-2)} i H\right\} .
\end{aligned}
$$

It is now evident that (4) has

$2(n-2)$ triple roots - the values for which $H=0$;

$3(n-2)$ double roots - the values for which $\tilde{t}=0$;

$n$ double roots - the values for which $f=0$;

$4(n-3)$ double roots - the values for which $(n-3)$ jf $-(2 n-3) i H=0$.

The values of $J$ giving the branch points of the Riemann surface are determined from the two following equations which are used instead of (4):

$$
\begin{gathered}
J f^{2}(3 i H-2 j f)=6 H^{3}, \\
(J-1)(3 i H-2 j f) f^{2}=-12 \tilde{t^{2}} .
\end{gathered}
$$

Then if 


$$
\begin{gathered}
H=0, \quad J=0 ; \\
\tilde{t}=0, \quad J=1 ; \\
f=0, \quad J=\infty ; \\
(n-3) j f-(2 n-3) i H=0, \\
J=\frac{6(3-n) H^{2}}{(n+3) i f^{2}}=\frac{6(3-n)^{3} j^{2}}{(n+3)(2 n-3)^{2} i^{3}} .
\end{gathered}
$$

The form of the equations $(4 a)$ and $(4 b)$ shows that all the sheets of the $6(n-2)$-sheeted Riemann surface come together, $k$ at a time, over the branch points $J=0$ and $J=1$ but do not do so over the remaining branch points.

6. The condition for the regularity of the Riemann surface at $J=\infty$ can be determined. The desirability of applying such a condition is obvious when the following theorem has been proved: If the Riemann surface is regular over $J=\infty, \tau$ is an elliptic modular function.

From (4) $J$ is a uniform function of $\tau$ and of $\omega$. By hypothesis the only critical points occur at $J=0,1, \infty$. But these give the vertices of the two nets of triangles obtained by mapping the real $J$ axis on the $\tau$ and $\omega$ planes respectively.* The positive half of the $J$ plane is mapped into a region within a triangle having one vertex at $\infty$ and the angles at the finite vertices equal to $\pi / 2$ and $\pi / 3$ respectively. This is true in both the $\tau$ and $\omega$ planes. Since a circuit around $J=0$ necessitates $\omega(0)=\omega_{0} \dagger$ and $\tau(0)=\tau_{0} \ddagger$ the two power series for $J$ as a function of $\tau$ and of $\omega$ are

$$
\begin{aligned}
& J=a_{0}\left(\omega-\omega_{0}\right)^{3}\left\{1+P\left(\omega-\omega_{0}\right)\right\}, \\
& J=b_{0}\left(\tau-\tau_{0}\right)^{3}\left\{1+P\left(\tau-\tau_{0}\right)\right\} .
\end{aligned}
$$

Therefore $\tau$ is a uniform function of $\omega$ at $\omega_{0}$; in fact

$$
\tau-\tau_{0}=\sqrt[3]{\frac{a_{0}}{b_{0}}}\left(\omega-\omega_{0}\right)\left\{1+P\left(\omega-\omega_{0}\right)\right\} .
$$

In like manner it is a uniform function of $\omega$ when $J=1$. The first term of the series will be of the form

$$
\tau-\tau_{1}=\sqrt{\frac{a_{1}}{b_{1}}}\left(\omega-\omega_{1}\right)
$$

where $\tau_{1}$ and $\omega_{1}$ are the values of $\tau$ and $\omega$ respectively for $J=1$. A circuit around $J=\infty$ leads into the negative half-plane. This is not mapped in

${ }^{*}$ K. F., I, p. $45 \mathrm{ff}$.

$\dagger$ K. F., I, p. 128.

$\ddagger(4)$. 
the given region of the $\tau$ and $\omega$ plane but in the reflection of that region. Therefore in the map of the positive or negative half of the $J$ plane, $\tau$ is a uniform function of $\omega$ and the theorem is proved.

But the condition that the Riemann surface be regular over $J=\infty$ is not yet known. In order to determine when all the sheets come together $k$ at a time, assume that $f$ and $H$ have no common roots and hence that $f$ has no double root. The form of $\left(H^{3}, \tilde{t}\right)^{1}$ shows that $f=0$ accounts for $2 n$ sheets. Hence there are $6(n-2)-2 n=4(n-3)$ sheets to be brought together over $J=\infty$ for the roots of $(n-3) j f-(2 n-3) i H=0$. But the corresponding form of $J$ shows that only when $i$ or $f$ vanishes can $J=\infty$.

Let the values $\left(\mu_{\tau}^{p}\right)^{k} \equiv p^{k}=0$ be those not included by $f=0$, which give the branch point at $J=\infty$. The order of the branch point is then $k-1$. Since the surface is to be regular

and

$$
3 i H-2 j f \approx f^{k-2} p^{k}, *
$$

$$
(n-3) j f-(2 n-3) i H \approx f^{k-2} p^{k-1} q,
$$

where $q$ refers to any other possible branch points. Since $p$ is to determine $J=\infty$,

$$
i=0 p h
$$

where $h$ is the result of dividing $i$ by $p$. Consider the order in the variables on each side of (5). Then

$$
4(n-3)=n(k-2)+k p, .
$$

where $p$ indicates the order of the form $p$. If $k=2, p=2(n-3)$. Therefore the form $p$ cannot be a factor of $i$ whose degree is $2(n-4)$, unless $i$ vanishes identically. If $k=3$, since $H$ by hypothesis cannot contain $f$ as a factor, $i$ must do so, because of $(5)$; i. e., $i==f p h^{\prime}$ where $h^{\prime}$ is the result of dividing $h$ by $f$. But when $k=3$, from (6) $p=n-4$. Hence

$$
2(n-4)=n+n-4+h^{\prime},
$$

where $h^{\prime}$ indicates the order of the form $h^{\prime}$. This is obviously impossible. In fact since $i H$ contains a factor $f^{k-2}$ (vide (5)), and $H$ contains no factor $f$, $i$ must contain the factor $f^{k-2}$. Therefore

$$
2(n-4)=p+n(k-2)+h^{\prime} .
$$

If $k>3, p+h^{\prime} \equiv-8$, again an impossibility, unless $i \equiv 0$. If $i \equiv 0$, then from (4a) $J=\infty$ when $j f^{3}=0$. But in the case under discussion $J$ can equal infinity only when $i$ or $f$ vanishes. Hence $j$ is either a constant or some power, $l$, of $f$; i. e.,

\footnotetext{
* The symbol $\approx$ is used to indicate equivalence of forms to within a multiplicative constant.
} 
or

$$
3(n-4)=\ln
$$

$$
n(3-l)=12 \text {. }
$$

If $l=0,1,2$, in turn, $n=4,6,12$. For the nics, where $n$ assumes only these values, the tetrahedral, octahedral, and icosahedral forms are obtained, when the condition $i \equiv 0$ is applied. On the other hand it is known* that in the case of the elliptic modular equations, the branch points of the Riemann surface can occur only at $J=0,1, \infty$.

The conclusions of the preceding paragraphs can be summarized as follows: If the Riemann surface on which (4) is mapped is made regular, then $i \equiv 0$, $\tau$ is an elliptic modular function, $f$ is a form associated with one of the regular bodies, and (4) is the corresponding modular equation.

The dihedral case has not yet been shown to be included in (4) but can easily be developed. Let

Then

$$
f \equiv\left(\alpha_{1} \tau_{1}+\alpha_{2} \tau_{2}\right)^{n} \equiv \tau_{1}^{n}-\tau_{2}^{n} .
$$

$$
\begin{aligned}
H & =-2 \tau_{1}^{n-2} \tau_{2}^{n-2}, \\
\tilde{t} & =-\tau_{1}^{n-3} \tau_{2}^{n-3}\left(\tau_{1}^{n}+\tau_{2}^{n}\right), \\
i & =-2 \tau_{1}^{n-4} \tau_{2}^{n-4}, \\
j & \equiv 0 .
\end{aligned}
$$

The syzygy (4) becomes

$$
J: J-1: 1=-4 \tau_{1}^{n} \tau_{2}^{n}:-\left(\tau_{1}^{n}+\tau_{2}^{n}\right)^{2}:\left(\tau_{1}^{n}-\tau_{2}^{n}\right)^{2} .
$$

This can easily be identified with the form of the dihedral equation given by Klein, $\dagger$ if the substitution $J=1 / z$ is made.

\section{The Elliptic NORM CURVE OF THIRd ORDER, $Q_{3}$, IN TWO-DIMENSIONAL SPACE, $S_{2}$}

7. Since the general configuration of the elliptic norm curve of third order $Q_{3}$ in two-dimensional space $S_{2}$, is familiar, it will be discussed in this paper only as it offers an opportunity of giving a geometric significance to some of the algebraic results previously obtained.

According to the work of Klein and Bianchi, $Q_{3}$, whose three homogeneous coördinates can be expressed as products of sigma functions, is transformed into itself by the group $G_{18}$ of collineations which send the space $S_{2}$ into itself. $\ddagger$ The normal form of its equation can be written

$$
x_{0}^{3}+x_{1}^{3}+x_{2}^{3}-3 \tau x_{0} x_{1} x_{2}=0 \text {. }
$$

${ }^{*}$ K. F., I, p. 574, § 1 .

$\dagger$ Klein, Vorlesungen über das Ikosaeder, p. 60.

$\ddagger$ K. F., II, pp. 246-256. 
In the coördinate system used, the reference triangle is an inflexional triangle. Since $\tau$ is defined by (7) in terms of a ratio of the $x$ 's, $\tau$ is an elliptic modular function, i. e., a function of $\omega_{1} / \omega_{2}$, where $\omega_{1}$ and $\omega_{2}$ are the periods of the sigma functions in terms of which $x_{i}$ is expressed.*

If in (7) $\tau$ is considered a parameter, then (7) represents a pencil of cubics on the nine inflexions. The degenerate curves of the pencil are the inflexional triangles and they intersect the proper curves at the inflexions of the proper curves.

8. An irrationality of the type discussed in section I occurs if one of the involutory $\dagger$ collineations in $G_{18}$ is considered. One such collineation is $x_{0}(-u)=x_{0}(u), x_{1}(-u)=x_{2}(u), x_{2}(-u)=x_{1}(u)$, where the center is the inflexion $(0,1,-1)$ and the axis upon which $Q_{3}$ is projected is the harmonic polar $x_{1}-x_{2}=0$. A pencil of lines

$$
t_{1} x_{0}+t_{2}\left(x_{1}+x_{2}\right)=0
$$

on the center establishes a two-to-one correspondence between the points of $Q_{3}$ and the points on the axis. The irrationality separating two corresponding points of $Q_{3}$ is from (7) and (8) found to be

$$
\frac{x_{1}}{x_{2}}=\frac{t^{3}-3 \tau t^{2}+2 \pm \sqrt{-3 t^{2}\left(t^{4}+2 \tau t^{3}-3 \tau^{2} t^{2}-4 t+4 \tau\right)}}{2\left(t^{3}-1\right)}\left(t=t_{1} / t_{2}\right) .
$$

Let

$$
t^{4}+2 \tau t^{3}-3 \tau^{2} t^{2}-4 t+4 \tau=(t-\tau)\left(t^{3}+3 \tau t^{2}-4\right) \equiv a_{t}^{4} .
$$

The values of $t$ for which $a_{t}^{4}=0$ give the double points of the involution, which are the points of contact of the tangents drawn from the inflexion. Since one of these tangents is the flex tangent which has the parameter $t=\tau$, $a_{t}^{4}$ can be written in the factored form given. The four flex lines on the point $(0,1,-1)$ have parameters given by

$$
\alpha_{t}^{4} \equiv t_{2}\left(t_{1}^{3}-t_{2}^{3}\right)=0
$$

an equianharmonic quartic. If $\alpha_{t}^{4}$ is polarized with respect to $\tau$, it becomes evident that $a_{t}^{4}=(t \tau) \alpha_{\tau} \alpha_{t}^{3}$. Hence the irrationality $\sqrt{a_{t}^{4}}$ is of exactly the type given in section I. A geometric interpretation of the result is that at an inflexion $I$ of $Q_{3}$, the three tangents from $I$ other than the flex tangent are given by the first polar of the flex tangent at $I$ as to the four flex lines on $I$.

* B., section 1. By using $\tau=-2 \tau$ in (7) Bianchi's form is obtained.

$\dagger$ †egre: Remarques sur les transformations uniformes des courbes elliptiques en elles-mêmes, Mathematis che Annalen, vol. 27, 1886.

$\ddagger$ The inflexions of $Q_{3}$ can be permuted in 216 different ways. If one is fixed as in the present case, the four flex lines on it can at the most be permuted among themselves. It is therefore to be expected that their parameters form an equianharmonic quartic invariant under a tetrahedral group. 
For the irrationality $\sqrt{a_{t}^{4}}$

$$
g_{2}=\frac{3 \tau}{4}\left(\tau^{3}+8\right), \quad g_{3}=\frac{1}{8}\left(\tau^{6}-20 \tau^{3}-8\right), \quad \Delta=-27\left(1-\tau^{3}\right)^{3} .
$$

The syzygy (4) becomes

$$
J: J-1: 1=\tau^{3}\left(\tau^{3}+8\right)^{3}:\left(\tau^{6}-20 \tau^{3}-8\right)^{2}: 64\left(\tau^{3}-1\right)^{3},
$$

the form of the modular equation* given by Klein. Hence $\tau$ is the tetrahedral irrationality. $\dagger$

III. The ELLIPTIC NORM CURVE OF FOURTH ORDER, $Q_{4}$, IN THREE-DIMENSIONAL SPACE, $S_{3}$

9. $\ddagger$ The coördinates of a point on the elliptic norm curve of fourth order, $Q_{4}$, in three-dimensional space $S_{3}$ are given by

$$
x_{a}(u)=c_{a} \prod_{\mu=0}^{3} \sigma_{a / 4+1 / 2,(\mu+1 ; 2) / 4}\left(u \mid \omega_{1}, \omega_{2}\right) \quad(\alpha=0, \cdots 3),
$$

where $c_{a}$ is an undetermined factor and

$$
\sigma_{a / 4, \mu / H}\left(u \mid \omega_{1}, \omega_{2}\right) \equiv e^{\left[\left(\lambda \eta_{1}+\mu \eta_{2}\right) / 4\right]\left[u-\left(\lambda \omega_{1}+\mu \omega_{2}\right) / 8\right]} \sigma\left(u-\frac{\lambda \omega_{1}+\mu \omega_{2}}{4} \mid \omega_{1}, \omega_{2}\right) .
$$

To obtain a convenient expression for $c_{a}$, Klein introduces the transformed periods $\bar{\omega}_{1}, \bar{\omega}_{2}$, such that $\bar{\omega}_{1}=\omega_{1}, \bar{\omega}_{2}=\omega_{2} / 4$. Because of the Legendre relation $\omega_{1} \eta_{2}-\omega_{2} \eta_{1}=2 \pi i$, the corresponding substitution for $\eta$ is $\bar{\eta}_{1}=4 \eta_{1}$, $\bar{\eta}_{2}=\eta_{2}$. The coördinates are finally given in the form

$x_{a}\left(u \mid \omega_{1}, \omega_{2}\right)=a_{a} e^{-\left[\left(\eta_{1}-4 \eta_{1}\right) / 2 \omega_{1}\right] u^{2}+\left([(a+2) / 4] \bar{\eta}_{1}+\bar{\eta} / 2\right) u}$

$$
\cdot e^{-\left([(a+2) / 4] \bar{\eta}_{1}+\bar{\eta}_{2} / 2 \times[(a+2) \cdot 8] \omega_{1}+\omega_{2} / 16\right)}
$$

$$
\cdot \sigma\left(u-\frac{\alpha+2}{4} \omega_{1}-\frac{\mu+\frac{1}{2}}{4} \omega_{2} \mid \omega_{1}, \frac{\omega_{2}}{4}\right),
$$

where

$$
a_{\alpha}=e^{-1 / 2[(\pi i a) / 4+(5 \pi i) / 2]} \mathrm{X},
$$

$\mathrm{X}$ being a function of the periods, independent of $u$ and $\alpha$. The notation indicates that $x_{a}(u)$ depends upon the periods $\omega_{1}, \omega_{2}$, the $\sigma$ functions upon the periods $\omega_{1}, \omega_{2} / 4$. In the coördinate system used $x_{a+4}(u)=x_{a}(u)$.

Four points $u_{1}, u_{2}, u_{3}, u_{4}$, of $Q_{4}$ are on a plane if

$$
u_{1}+u_{2}+u_{3}+u_{4} \equiv 0 \quad\left(\bmod \omega_{1}, \omega_{2}\right) .
$$

* K. F., I, p. 104.

† See B., section 1, for further discussion of $\tau$.

$\$ \$ \$ 9$ and 10 do little more than give for the case $n=4$ the general theory stated by Klein for $Q_{n}$ (K. F., II, pp. 261-265). 
There are 16 singular points (points of hyperosculation), which lie by fours on the faces of a tetrahedron $T$. These sets of fours are

$$
\frac{1}{2} P, \quad \frac{\omega_{1}}{4}+\frac{1}{2} P, \quad \frac{\omega_{2}}{4}+\frac{1}{2} P, \quad \frac{\omega_{1}+\omega_{2}}{4}+\frac{1}{2} P,
$$

where

$$
\frac{1}{2} P=0, \quad \frac{\omega_{1}}{2}, \quad \frac{\omega_{2}}{2}, \quad \frac{\omega_{1}+\omega_{2}}{2} .
$$

10. The curve $Q_{4}$ admits a collineation group $G_{32}$ determined by the 32 substitutions

$$
u^{\prime}= \pm u+{\frac{p \omega_{1}+q \omega_{2}}{4}}^{*} \quad(p, q=0,1,2,3) .
$$

Under $G_{32}, Q_{4}$ is invariant; and under (11), (9) is invariant. The $G_{32}$ is defined abstractly by $K_{1}, K_{2}, K_{3}$, such that

$$
\begin{aligned}
K_{1}^{2}=K_{2}^{4} & =K_{3}^{4}=1, & & K_{2} K_{3}=K_{3} K_{2}, \\
K_{1} K_{2} & =K_{2}^{3} K_{1}, & & K_{1} K_{3}=K_{3}^{3} K_{1} .
\end{aligned}
$$

These generators can be taken to be

$$
\begin{aligned}
& u^{\prime}=-u, \quad u^{\prime}=u-\frac{\omega_{1}}{4}, \quad u^{\prime}=u-\frac{\omega_{2}}{4}, \quad \text { i. e., } \\
& x_{a}(-u)=x_{4-a}(u), \quad x_{a}\left(u-\frac{\omega_{1}}{4}\right)=x_{a+1}(u), \\
& x_{a}\left(u-\frac{\omega_{2}}{4}\right)=\epsilon^{a} x_{a}(u), \dagger \quad\left(\epsilon=e^{(2 \pi i) / 4}\right) .
\end{aligned}
$$

The $G_{32}$ contains an invariant Abelian $G_{16}$ generated by $K_{2}$ and $K_{3}$. This $G_{16}$ contains three pairs of cyclic $G_{4}$ 's, each pair having a common element of period two. The three elements of period two, $K_{2}^{2}, K_{3}^{2}, K_{2}^{2} K_{3}^{2}$, and identity constitute a four-group whose parametric expression is

$$
u^{\prime}=u+\frac{1}{2} P .
$$

By adjoining $K_{1}$ four new elements of period two, which with the four-group form an Abelian $G_{8}$, are obtained. Their parametric expression is

$$
u^{\prime}=-u+\frac{1}{2} P \text {. }
$$

From (10) they are evidently reflexions in vertex and opposite plane of $T$. Thus the unique tetrahedron $T$ can be determined from the $K$ 's. By the transformations

$$
y_{0}=x_{0}+x_{2}, \quad y_{1}=x_{1}+x_{3}, \quad y_{2}=x_{0}-x_{2}, \quad y_{3}=x_{1}-x_{3},
$$

* K. F., II, pp. 237-242; and Segre, loc. cit.

$\dagger$ For factors of proportionality see K. F., II, p. 264. 
$T$ is made the reference tetrahedron and $K_{1}, K_{2}, K_{3}$ take the forms

$$
\begin{array}{cc}
C_{1} & C_{2} \\
y_{0}(-u)=y_{0}(u) & y_{0}\left(u-\frac{\omega_{1}}{4}\right)=y_{1}(u) \\
y_{1}(-u)=y_{1}(u) & y_{1}\left(u-\frac{\omega_{1}}{4}\right)=y_{0}(u) \\
y_{2}(-u)=y_{2}(u) & y_{2}\left(u-\frac{\omega_{1}}{4}\right)=y_{3}(u) \\
y_{3}(-u)=-y_{3}(u) & y_{3}\left(u-\frac{\omega_{1}}{4}\right)=-y_{2}(u) \\
y_{0}\left(u-\frac{\omega_{2}}{4}\right) & =y_{2}(u) \\
y_{1}\left(u-\frac{\omega_{2}}{4}\right) & =i y_{3}(u) \\
y_{2}\left(u-\frac{\omega_{2}}{4}\right) & =y_{0}(u) \\
y_{3}\left(u-\frac{\omega_{2}}{4}\right) & =-i y_{1}(u) .
\end{array}
$$

11. The pencil of quadrics on $Q_{4}$ has a unique self-polar tetrahedron whose vertices are the vertices of cones on $Q_{4}$. Since the lines joining corresponding points $u,-u$, and $v,-v$ under $u^{\prime}=-u$ intersect, this tetrahedron is evidently $T$. Thus $G_{32}$ leaves the pencil of quadrics unaltered and permutes the four cones under a four-group, which is the factor group of $G_{32}$ with reference to the $G_{8}$ mentioned in $\S 10$.

If the cone with vertex opposite $y_{3}=0$ is

$$
a y_{0}^{2}+b y_{1}^{2}+c y_{2}^{2}=0,
$$

the others furnished by the four-group are

These are in a pencil if

$$
\begin{gathered}
b y_{0}^{2}+a y_{1}^{2}+c y_{3}^{2}=0, \\
c y_{0}^{2}+a y_{2}^{2}-b y_{3}^{2}=0, \\
c y_{1}^{2}-b y_{2}^{2}+a y_{3}^{2}=0 . \\
a^{2}=b^{2}+c^{2}
\end{gathered}
$$

or if

Trans. Am. Math. Soc. 18 


$$
a=\tau_{1}^{2}+\tau_{2}^{2}, \quad b=\tau_{1}^{2}-\tau_{2}^{2}, \quad c=2 \tau_{1} \tau_{2} .
$$

As $\tau$ varies the family of $Q_{4}$ 's, each of which admits the same $G_{32}$, is obtained. This family lies on the surface

$$
y_{0}^{4}-y_{1}^{4}-y_{2}^{4}+y_{3}^{4}=0
$$

obtained by eliminating $\tau$.

12. Let $P_{0} \cdots P_{3}$ be the vertices of $T ; L_{i}=\overline{P_{0} P_{i}}, L_{i}^{\prime}=\overline{P_{j} P_{k}},(i, j, k$ $=1,2,3)$, pairs of opposite edges of $T$. Let a cyclic $G_{4}(\$ 10)$ be generated by $C_{2}$ or $u^{\prime}=u-\omega_{1} / 4$. Then $C_{2}$ is not perspective, for if it had a pencil of fixed planes, the pencil would cut the curve in sets of four conjugate co-planar points, whereas the general set $u, u-\omega_{1} / 4, u-2 \omega_{1} / 4$, $u-3 \omega_{1} / 4$ is not coplanar. Hence $C_{2}$ has a fixed tetrahedron $T_{i}$ whose vertices are on a line pair $L_{i}, L_{i}^{\prime}$ of $T$, since $C_{2}^{2}$ is a reflexion in such a line pair. The reflexions in the other line pairs leave the cyclic $G_{4}$ unaltered, whence the vertices of $T_{i}$ on $L_{i}$ and $L_{i}^{\prime}$ are harmonic with those of $T$. The second cyclic $G_{4}$ containing $C_{2}^{2}(\S 10)$ has a fixed tetrahedron $T_{i}^{\prime}$. Thus the vertices of $T, T_{i}, T_{i}^{\prime}$ on $L_{i}, L_{i}^{\prime}$ form three mutually harmonic pairs on each line $L_{i}$. Since the cyclic $G_{4}$ 's are invariant under $G_{32}$, the six tetrahedra $T_{i}, T_{2}^{\prime}$ are invariant under $G_{32}$. The edges of the six tetrahedra $T_{i}, T_{i}^{\prime}$, exclusive of the edges common to $T$, form six skew quadrilaterals.

The elements of $G_{32}$ not in $G_{16}$ are involutory. The four of type

$$
u^{\prime}=-u+\frac{1}{2} P
$$

are reflexions in vertex and opposite plane of $T$ (vide $\$ 10$ ). The remaining twelve are $u^{\prime}=-u+\frac{1}{4} P\left(\frac{1}{4} P \neq \frac{1}{2} P\right)$. Since two pairs of corresponding points of $Q_{4}$ are not in general coplanar, these twelve collineations are reflexions in skew line pairs. If $u^{\prime}=-u+\frac{1}{4} P$ is a reflexion in the line pair $M_{i}, M_{i}^{\prime}$, then, since it transforms $u^{\prime}=-u+\frac{1}{2} P^{\prime}$ into $u^{\prime}=-u+\frac{1}{2} P+\frac{1}{2} P^{\prime}$, it must interchange the vertices of $T$, i. e., $M_{i}, M_{i}^{\prime}$ lie across two skew edges of $T$ say $L_{i}$ and $L_{i}^{\prime}$. The product of $u^{\prime}=-u+\frac{1}{4} P$ and $u^{\prime}=u+\frac{1}{2} P$, i. e., $u^{\prime}=-u+\frac{3}{4} P$ will be another of the twelve involutions whose line pair $N_{i}, N_{i}^{\prime}$ will cross both $L_{i}, I_{i}^{\prime}$ and $M_{i}, M_{i}^{\prime}$. Thus the twelve involutions determine six pairs of line pairs which form six skew quadrilaterals $\bar{T}_{i}$ with vertices on $L_{i}, L_{i}^{\prime}$. These six quadrilaterals separate into three pairs $\bar{T}_{i}, \bar{T}_{i}^{\prime}$, the two of a pair having their vertices on the same line pair $L_{i}, L_{i}^{\prime}$.

The involution on $M_{i}, M_{i}^{\prime}$ leaves unaltered the line $L_{i}$ and the tetrahedra $T_{i}, T_{i}^{\prime}$. Since it cannot interchange all three pairs of vertices of $T_{i}, T_{i}^{\prime}, T$ on $L_{i}$ the pair must cross $L_{i}$ at the vertices of $T_{i}$ or $T_{i}^{\prime}$. Hence: The six skew quadrilaterals $T_{i}, T_{i}^{\prime}$ and the six skew quadrilaterals $\bar{T}_{i}, \bar{T}_{i}^{\prime}$ have the same vertices.

If $a_{i}, b_{i}$ are the pairs of vertices on $L_{i}, a_{i}^{\prime}, b_{i}^{\prime}$ the pairs on $L_{i}^{\prime}$, and if the 
quadrilaterals $T_{i}, T_{i}^{\prime}$ are formed by joining $a_{i}$ to $a_{i}^{\prime}, b_{i}$ to $b_{i}^{\prime}$, then the quadrilaterals $\bar{T}_{i}, \bar{T}_{i}^{\prime}$ are formed by joining $a_{i}$ to $b_{i}^{\prime}, b_{i}$ to $a_{i}^{\prime}$.

13. If an elliptic quartic degenerates, at least two of the four cones on it must coincide. From equations (13)-(16) it can be seen that this requires $a$ or $b$ or $c$ to be zero. Then two pairs of cones each coincide into pairs of planes and the quartic is a skew quadrilateral. There are six such $Q_{4}$ 's in the family, corresponding to the values of the modulus given by

$$
\alpha_{\tau}^{6} \equiv \tau_{1}^{5} \tau_{2}-\tau_{1} \tau_{2}^{5}=0 .
$$

On the other hand, the six quadrilaterals $T_{i}$ admit $G_{32}(\$ 12)$ and occur in the family. Therefore the quadrilaterals $T_{i}$ are on the quartic surface (19).

The parametric representation shows that the involution on $M_{i}, M_{i}^{\prime}$ bas four fixed points on each $Q_{4}$. This line pair meets each $T_{i}$ in four points. It has been shown above that for $T_{i}$ and $T_{i}^{\prime}$ these four points are two vertices or double points. For $T_{j}, T_{j}^{\prime}$ the four points are paired on opposite edges. Thus $M_{i}$ has more than four points in common with the surface (19) and therefore lies on it. But the surface has just 48 lines determined by the separation of its terms into two sets of two. Hence the twelve skew quadrilaterals $T_{i}, T_{i}^{\prime} ; \bar{T}_{i}, \bar{T}_{i}^{\prime}$ are the 48 lines of the quartic locus of the family of $Q_{4}$ 's which admit the given $G_{32}$. The six quadrilaterals $T_{i}, T_{i}^{\prime}$ are the degenerate curves of the family.*

14. In order to introduce the irrationality discussed in section I, the family of $Q_{4}$ 's will be projected into a family of doubly-covered rational curves as was done in the case of $Q_{3}$ (section II). In that simple case the projected family lay on a single line. The projection was made by means of one of the involutions which belonged to every curve. In the present case there are two types of involution in $G_{32}$ which are not in $G_{16}$. By using the type determined by a vertex and opposite plane of $T$, the family of $Q_{4}$ 's is projected upon a family of doubly-covered conics. By using the type determined by two skew lines of the quadrilateral $\bar{T}_{i}$ the projection is upon a family of doubly covered lines all of which lie on one line of the pair.

For the first type, if $y_{3}=0$ is the plane of the projection, the family of doubly-covered conics is from (13)

$$
a y_{0}^{2}+b y_{1}^{2}+c y_{2}^{2}=0 \text {. }
$$

In order to introduce a parameter $t$ on the conic, which is covariantly related to the modulus $\tau$ of the quartic, it is noted that the reference triangle cuts out on the conic an octahedral sextic which can be identified with $\alpha_{\tau}^{6}$, the one which determines the degenerate curves. Then the conic can be written as follows:

* Segre, loc. cit., and Concerning the twisted biquadratic, by J. C. Kluyver, A m e r i c a $\mathbf{n}$ J ournal of Mathematics, vol. 19 (1897), p. 319 ff. 
and from (14)

$$
\begin{aligned}
& y_{0}=\sqrt{\tau_{1}^{2}-\tau_{2}^{2}} \sqrt{2 \tau_{1} \tau_{2}}\left(t_{1}^{2}+t_{2}^{2}\right), \\
& y_{1}=i \sqrt{2 \tau_{1} \tau_{2}} \sqrt{\tau_{1}^{2}+\tau_{2}^{2}}\left(t_{1}^{2}-t_{2}^{2}\right), \\
& y_{2}=i \sqrt{\tau_{1}^{2}+\tau_{2}^{2}} \sqrt{\tau_{1}^{2}-\tau_{2}^{2}}\left(2 t_{1} t_{2}\right),
\end{aligned}
$$

$$
y_{3}= \pm 2 \sqrt{2(t \tau) \alpha_{\tau}^{3} \alpha_{\tau}^{3}} .
$$

The geometric interpretation of the radical in $t$ can be stated thus: If an elliptic quartic lies on a cone, the six generators on pairs of points of hyperoscuJation form an octahedral sextic, and the four generators on single points of hyperosculation are such that any three form the cubic polar of the remaining one as to the sextic.

The expression for $\tau=\tau_{1} / \tau_{2}$ as an elliptic modular function can be obtained from (21) and (19) in the form

$$
\tau=\frac{\tau_{1}}{\tau_{2}}=\frac{-y_{2}^{2}+\sqrt{y_{2}^{4}-y_{0}^{4}+y_{1}^{4}}}{y_{0}^{2}+y_{1}^{2}}=\frac{-y_{2}^{2}+y_{3}^{2}}{y_{0}^{2}+y_{1}^{2}} \text {. }
$$

If this is evaluated for $u=0$ and expressed in terms of $x_{i}$ (vide $\S 10$ ), the following expression is obtained

$$
\tau=\frac{-\left[x_{0}(0)-x_{2}(0)\right]^{2}+\left[x_{1}(0)-x_{3}(0)\right]^{2}}{\left[x_{0}(0)+x_{2}(0)\right]^{2}+\left[x_{1}(0)+x_{3}(0)\right]^{2}} .
$$

The modular equation satisfied by $\tau$ can be obtained by forming $g_{2}, g_{3}$, and $\Delta$ for the irrationality $\sqrt{2(t \tau) \alpha_{\tau}^{3} \alpha_{t}^{3}}$ as in section I by letting

$$
n=6, \quad \alpha_{t}^{6}=t_{1}^{5} t_{2}-t_{1} t_{2}^{5} .
$$

The equation appears in the well-known octahedral form.*

The presence of the radicals $\sqrt{a}, \sqrt{b}, \sqrt{c}$ in the parametric form (22) of $Q_{4}$ is due to the fact that in the plane $y_{3}=0$ there are four points of hyperosculation determined by $t= \pm \tau, \pm 1 / \tau$, the roots of $(t \tau) \alpha_{\tau}^{3} \alpha_{t}^{3}=0$. One of these, $t=\tau$, has been isolated by the choice of the parameter $t$.

A striking form of the elliptic integral can be deduced from the type

$$
u=\int \frac{(t d t)}{\sqrt{(t \tau) \alpha_{\tau}^{3} \alpha_{t}^{3}}} .
$$

The family of $Q_{4}$ 's has been projected into the family of conics (21) which are intimately related to the quartic section of the family of $Q_{4}$ 's, made by the plane of projection. This quartic section $\dagger$ is

\footnotetext{
* K. F., I, p. 20.
}

$\dagger$ Uber Untersuchung und Aufstellung von Gruppe und Irrationalität regulärer Riemann'schen Flächen and Notiz über eine reguläre Riemann'sche Fläche vom Geschlechte drei und die zugehörige "Normalcurve" vierter Ordnung, by W. Dyck in M a the matis che Annalen, vol. 17 (1880), pp. 473 and 510. The connection is here made with the elliptic modular function, but the system of conics mentioned above is not considered. The normal forms of the elliptic integral are to be contrasted with those described in an article by Klein in the same volume. 
or parametrically

$$
a_{y}^{4} \equiv y_{0}^{4}-y_{1}^{4}-y_{2}^{4}=0
$$

$$
y_{0}=\sqrt{a}, \quad y_{1}=i \sqrt{b}, \quad y_{2}=i \sqrt{c} .
$$

The family (21) is evidently the system of polar conics as to $a_{y}^{4}$, of a point $y$ on $a_{y}^{4}=0$. Moreover $a_{y}^{4}=0$ is the envelope of this quadratic family of conics (21), which appears therefore as an isolated Steiner system of the quartic curve.

Consider the integral

$$
I=\int \frac{(y x x d)}{\sqrt{a_{y}^{3} a_{x} \cdot\left(a a^{\prime} a^{\prime \prime}\right) a_{y}^{3}\left(a^{\prime} a^{\prime \prime} y x\right)^{3}}},
$$

where $x$ and $y$ are subject to the relations $a_{y}^{4}=0, a_{y}^{2} a_{x}^{2}=0$. The integral $I$ depends first upon the choice of the point $y$ on $a_{y}^{4}=0$, secondly upon $x_{0}: x_{1}: x_{2}$, a point which is required to be on (21), $a_{y}^{2} a_{x}^{2}=0$. That $I$ is an elliptic integral follows from the fact that if a parameter is introduced on the rational curve the radical reduces to one of degree four. This can easily be verified. Let

$$
a_{1}=t_{1}^{2}+t_{2}^{2}, \quad b_{1}=t_{1}^{2}-t_{2}^{2}, \quad c_{1}=2 t_{1} t_{2} .
$$

Then with $y$ expressed as in (24) and $a_{y}^{2} a_{x}^{2}=0$ as in (22) it is found that

Since

$$
(y x d x)=-4 \sqrt{a} \sqrt{b} \sqrt{c}(t \tau)^{2}(t d t) .
$$

$$
\begin{aligned}
\left(a^{\prime} a^{\prime \prime} u\right)^{4} & =2\left(u_{0}^{4}-u_{1}^{4}-u_{2}^{4}\right), \\
a_{y}^{3} a_{x} & =4\left(y_{0}^{3} x_{0}-y_{1}^{3} x_{1}-y_{2}^{3} x_{2}\right), \\
\left(a a^{\prime} a^{\prime \prime}\right) a_{y}^{3}\left(a^{\prime} a^{\prime \prime} u\right)^{3} & =32\left(y_{0}^{3} u_{0}^{3}+y_{1}^{3} u_{1}^{3}+y_{2}^{3} u_{2}^{3}\right) .
\end{aligned}
$$

Instead of the line coördinates introduce the values

$$
u_{0}=u_{1} x_{2}-y_{2} x_{1}=\sqrt{a}\left(-b c_{1}+b_{1} c\right)=\sqrt{a}(t \tau)\left(t_{1} \tau_{1}+t_{2} \tau_{2}\right) \text {, etc. }
$$

Then (25) becomes

$$
\left(a a^{\prime} a^{\prime \prime}\right) a_{y}^{3}\left(a^{\prime} a^{\prime \prime} u\right)^{3}=\frac{16}{5}(t \tau)^{3} \alpha_{\tau}^{3} \alpha_{t}^{3},
$$

and

$$
a_{y}^{3} a_{x}=8 \sqrt{a} \sqrt{b} \sqrt{c}(t \tau)^{2} .
$$

The integral $I$ can now be written

$$
I=-\frac{\sqrt{5}}{\sqrt[4]{a b c}} \int^{\bullet} \frac{(t d t)}{2 \sqrt{2(t \tau) \alpha_{\tau}^{3} \alpha_{t}^{3}}} .
$$

If $a_{y}^{4}=0$ is the Dyck quartic automorphic under a collineation group $G_{96}$, the integral 


$$
I=\int \frac{(y x d x)}{\sqrt{a_{y}^{3} a_{x} \cdot\left(a a^{\prime} a^{\prime \prime}\right)} \overline{a_{y}^{3}\left(a^{\prime} a^{\prime \prime} y x\right)^{3}}}
$$

is an elliptic integral of the first kind with moduli $y_{0}: y_{1}: y_{2}$, and variables $x_{0}: x_{1}: x_{2}$ on the polar conic of $y$ as to the quartic. This integral is unaltered if the variables and moduli are cogrediently transformed under $G_{96}$.

If $a_{y}^{4}=0$ is the Klein quartic invariant under the collineation group $G_{168}$ the integral I has the same properties and covariant character.

The last statement is given here without proof. The theorem indicates the possibility of extending the canonical form obtained for genus zero to larger values of the genus and gives the form applicable to genus three.

For the second type of projection mentioned in the beginning of this paragraph, the involution $C_{1} C_{2}$ or parametrically $u^{\prime}=-u+\omega_{1} / 4$ is used. A pencil of planes on one fixed line of $C_{1} C_{2}$ is given by

$$
y_{0}+y_{1}+i \sqrt{\tau} \lambda\left(y_{2}+y_{3}\right)=0 \text {. }
$$

By eliminating $y_{0} / y_{3}, y_{1} / y_{3}$ from (26), (13), and (14) the value of $y_{2} / y_{3}$ for the points of intersection of the plane (26) and $Q_{4}$ is found to be

$$
\frac{y_{2}}{y_{3}}=\frac{[a+b]\left[c^{2}-\tau^{2} \lambda^{4}(a-b)^{2}\right] \mp 2 \sqrt{\tau} \lambda c \sqrt{8(\lambda \tau) \alpha_{\tau}^{3} \alpha_{\lambda}^{3}}}{\tau^{2} \lambda^{4} c^{2}(a-b)-2 \tau \lambda^{2} c(a-b)^{2}+c^{2}(a+b)}
$$

The irrationality in (27) which separates corresponding points of $Q_{4}$ is again in the canonical form.*

15. Since $\tau$ is an elliptic modular function and the octahedral irrationality, it admits the group $\Gamma_{24}$ the principal subgroup of $\Gamma_{48}$, which is generated by

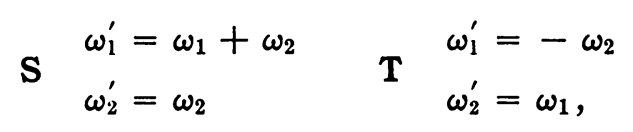

where the determinants of the transformations are congruent to identity $(\bmod 4)$. The effect of $\mathbf{S}$ and $\mathbf{T}$ on the coördinates of a point of $Q_{4}$ is given by

$$
\begin{array}{llll}
\mathbf{S}_{1}: y_{0}^{\prime}=\sqrt{i} y_{2}, & y_{1}^{\prime}=i y_{1}, & y_{2}^{\prime}=\sqrt{i} y_{0}, & y_{3}^{\prime}=i y_{3} \\
\mathbf{T}_{1}: y_{0}^{\prime}=y_{0}, & y_{1}^{\prime}=y_{2}, & y_{2}^{\prime}=y_{1}, & y_{3}^{\prime}=-i y_{3} ; \dagger
\end{array}
$$

on the modulus $\tau$, by

* The explicit equations of the quadrics generated by the lines joining corresponding points of $Q_{0}$ under $C_{1} C_{2}$ and the eleven other involutions of the same type can be derived (vide also Segre, loc. cit.). The parameters of these six quadrics as members of the pencil (13) $+\mu(14)$ $=0$ form an octahedral sextic.

$\dagger K$. F., II, p. 299, (12) and (13). There is a misprint in (12) but the form is given correctly on p. 297 . Klein gives the transformation on $x$ 's for $Q_{n}$. 


$$
\begin{array}{lll}
\mathbf{S}_{2}: \tau^{\prime}=\frac{\tau-i}{\tau+i} & \text { or } & \tau^{\prime}=\frac{\tau+i}{\tau-i}, \\
\mathbf{T}_{2}: \tau^{\prime}=\frac{\tau+1}{\tau-1} & \text { or } & \tau^{\prime}=\frac{\tau-1}{\tau+1} .
\end{array}
$$

The first substitutions of $S_{2}$ and $T_{2}$ are sufficient to generate the octahedral group $G_{24}$ on $\tau$.

By the addition of $G_{24}$ to $G_{32}$ a $G_{32.24}$ is obtained under which the quartics of the family are at the most permuted among themselves.

If now the surface

$$
y_{0}^{4}-y_{1}^{4}-y_{2}^{4}+y_{3}^{4}=0
$$

is considered, it will readily be seen that (19) is unaltered by the twenty-four permutations and the four letters $y_{i}$ combined with the roots of -1 , of which there are three choices. Hence a $G_{24 \cdot 4 \cdot 4 \cdot 4}$ leaves (19) unaltered. But a $G_{32 \cdot 24}$ has already been accounted for. The one type of transformation not yet included is

$C_{4}$

$$
y_{0}^{\prime}=y_{0}, \quad y_{1}^{\prime}=y_{1}, \quad y_{2}^{\prime}=y_{2}, \quad y_{3}^{\prime}=i y_{3} .
$$

The surface (19) is invariant under $C_{4}$ but its two sets of twenty-four lines are interchanged, $i$. e., the axes of the twelve involutory collineations of type $C_{1} C_{2}$ which form the skew quadrilaterals $\bar{T}_{i}, \bar{T}_{i}^{\prime}$, are transformed into the degenerate curves forming the skew quadrilaterals $T_{i}, T_{i}^{\prime}$ and vice versa.* This indicates the existence of two families of $Q_{4}$ 's on the surface (19) arranged as are the generators of a quadric in that through every point of the surface passes a curve of each family. But a curve of one family cuts every curve of the other family in eight points.

IV. The Elliptic NORM CURVE OF THE FIFTH ORDER, $Q_{5}$, IN FOUR-DIMENSIONAL SPACE, $S_{4}$

16. The reader is referred to the frequently cited article of Bianchi for the preliminary work needed in the discussion of the elliptic curve of fifth order, $Q_{5}$, in four-dimensional space $S_{4}$. In accordance with the notation of that article, the six fundamental pentahedra will be designated by $P_{\infty}, P_{i}$ ( $i=0$, $\cdots, 4)$. The collineations determined by the substitutions $u^{\prime}=-u$, $u^{\prime}=u-\omega_{1} / 5, u^{\prime}=u+\omega_{2} / 5$ will be represented by $C_{1}, C_{2}, C_{3}$ respectively. These are the generators of the fifty collineations, $G_{50}$, under which $Q_{5}$ is invariant. The five quadrics whose common intersection is $Q_{5}$ are

$$
\phi_{i} \quad \tau_{1} \tau_{2} x_{i}^{2}+\tau_{1}^{2} x_{i+2} x_{i+3}-\tau_{2}^{2} x_{i+1} x_{i+4}=0 \dagger \quad\left(i=0, \cdots 4 ; x_{i+6}=x_{i}\right) .
$$

* See also Kluyver, loc. cit.

† B., $\S 13$. The $a$ of Bianchi is replaced by $\tau_{1} / \tau_{2}$. 
In order to develop the elliptic irrationality it is convenient to introduce coördinates suggested by the involution $C_{1}$. Let

$$
\begin{gathered}
y_{0}=x_{0}, \quad y_{1}=x_{1}+x_{4}, \quad y_{2}=x_{2}+x_{3}, \\
z_{1}=x_{1}+x_{4}, \quad z_{2}=-x_{2}+x_{3} .^{*}
\end{gathered}
$$

Then $C_{1}$ has a plane $\pi$ of fixed points determined by $z_{1}=z_{2}=0$, in which the coördinates are $y_{0}: y_{1}: y_{2}$; and a skew line $L$ of fixed points determined by $y_{0}=y_{1}=y_{2}=0$, in which the coördinates are $z_{1}: z_{2}$. The explicit form of $C_{1}$ in the new coördinate system $\dagger$ is

$C_{1}^{\prime}: \quad y_{i}(-u)=-y_{i}(u), \quad z_{j}(-u)=z_{j}(u) \quad(i=0,1,2 ; j=1,2)$.

Since corresponding points in the involution are cut out by a pencil of $S_{3}$ 's on $\pi$, such points will have for their coördinates the same $y_{i}^{\prime}$ 's and the $z_{j}^{\prime}$ 's differing by the sign of a radical. The radical can easily be obtained.

17. The quadrics $\phi_{i}$ can be rewritten in terms of $y$ and $z$.

$$
\begin{array}{lrl}
\phi_{0}^{\prime} . & 4 \tau_{1} \tau_{2} y_{0}^{2}+\tau_{1}^{2} y_{2}^{2}-\tau_{2}^{2} y_{1}^{2}=\left(\tau_{1} z_{2}+\tau_{2} z_{1}\right)\left(\tau_{1} z_{2}-\tau_{2} z_{1}\right), \\
\phi_{1}^{\prime} . & \tau_{1} \tau_{2} y_{1}^{2}+\tau_{1}^{2} y_{1} y_{2}-2 \tau_{2}^{2} y_{0} y_{2}=\tau_{1} z_{1}\left(\tau_{1} z_{2}-\tau_{2} z_{1}\right), \\
\phi_{2}^{\prime} . & \tau_{1} \tau_{2} y_{2}^{2}+2 \tau_{1}^{2} y_{0} y_{1}-\tau_{2}^{2} y_{1} y_{2}=-\tau_{2} z_{2}\left(\tau_{1} z_{2}-\tau_{2} z_{1}\right), \\
\phi_{3}^{\prime} . & 2 \tau_{2}^{2} z_{2} y_{0}+\left(\tau_{1}^{2} z_{2}+2 \tau_{1} \tau_{2} z_{1}\right) y_{1}-\tau_{1}^{2} z_{1} y_{2}=0, \\
\phi_{4}^{\prime} . & 2 \tau_{1}^{2} z_{1} y_{0}+\tau_{2}^{2} z_{2} y_{1}+\left(\tau_{2}^{2} z_{1}+2 \tau_{1} \tau_{2} z_{2}\right) y_{2}=0 .
\end{array}
$$

Since $\phi_{3}^{\prime}$ and $\phi_{4}^{\prime}$ are homogeneous in $y_{i}$, they can be solved for $y_{i}$.

$$
\begin{aligned}
& y_{0}=-\rho \tau_{1} \tau_{2}\left[\tau_{2}^{2} z_{1}^{2}+3 \tau_{1} \tau_{2} z_{1} z_{2}+\tau_{1}^{2} z_{2}^{2}\right], \\
& y_{1}=\rho\left[\tau_{1}^{4} z_{1}^{2}+\tau_{2}^{4} z_{1} z_{2}+2 \tau_{1} \tau_{2}^{3} z_{2}^{2}\right], \\
& y_{2}=\rho\left[2 \tau_{1}^{3} \tau_{2} z_{1}^{2}+\tau_{1}^{4} z_{1} z_{2}-\tau_{2}^{4} z_{2}^{2}\right],
\end{aligned}
$$

where $\rho$ is an undetermined factor of proportionality. By substituting these values in $\phi_{0}^{\prime}, \phi_{1}^{\prime}, \phi_{2}^{\prime}$ the condition which $\rho$ must satisfy is found to be

$$
\begin{aligned}
\rho^{2}\left\{z_{1}^{3}\left[4 \tau_{1}^{3} \tau_{2}^{6}+3 \tau_{1}^{8} \tau_{2}\right]+z_{1}^{2} z_{2}\left[18 \tau_{1}^{4} \tau_{2}^{5}+\tau_{1}^{9}\right]+z_{1} z_{2}^{2}\left[18 \tau_{1}^{5} \tau_{2}^{4}-\tau_{2}^{9}\right]\right. \\
\left.+z_{2}^{2}\left[4 \tau_{1}^{6} \tau_{2}^{3}-3 \tau_{1} \tau_{2}^{8}\right]\right\}=\tau_{1} z_{2}-\tau_{2} z_{1} .
\end{aligned}
$$

Let $\alpha_{z}^{12} \equiv z_{1} z_{2}\left[z_{1}^{10}+11 z_{1}^{5} z_{2}^{5}-z_{2}^{10}\right]$. Then (28) can be written

$$
\begin{gathered}
4 \rho^{2} \alpha_{\tau}^{9} \alpha_{z}^{3}=(\tau z) . \\
\therefore \quad \rho=\frac{(\tau z)}{2 \sqrt{(\tau z) \alpha_{\tau}^{9} \alpha_{z}^{3}}} .
\end{gathered}
$$

* Whenever a collineation is indicated by an accented letter its form in terms of $y$ and $z$ is meant.

† K. F., II, p. 267 especially (13). 
If this value of $\rho$ is used, $Q_{5}$ can be written parametrically in terms of $z$.

$$
\begin{aligned}
& y_{0}=-\tau_{1} \tau_{2}(\tau z)\left[\tau_{2}^{2} z_{1}^{2}+3 \tau_{1} \tau_{2} z_{1} z_{2}+\tau_{1}^{2} z_{2}^{2}\right] \\
& y_{1}=(\tau z)\left[\tau_{1}^{4} z_{1}^{2}+\tau_{2}^{4} z_{1} z_{2}+2 \tau_{1} \tau_{2}^{3} z_{2}^{2}\right], \\
& y_{2}=(\tau z)\left[2 \tau_{1}^{3} \tau_{2} z_{1}^{2}+\tau_{1}^{4} z_{1} z_{2}-\tau_{2}^{4} z_{2}^{2}\right], \\
& z_{1}=2 z_{1} \sqrt{(\tau z) \alpha_{\tau}^{9} \alpha_{z}^{3}}, \\
& z_{2}=2 z_{2} \sqrt{(\tau z) \alpha_{\tau}^{9} \alpha_{z}^{3}} .
\end{aligned}
$$

Now consider a pencil of $S_{3}$ 's on $\pi$, given by

$$
t_{2} z_{1}=t_{1} z_{2} \text {. }
$$

If in (29) $z$ is replaced by $t,(29)$ gives the coördinates of pairs of points in the involution, which by (30) project into one point on $L$. The notation is then exactly analogous to that used in earlier sections of this paper. But $z$ can equally well be thought of as the variable parameter along a line. In either notation the irrationality which appears is of the type discussed in section I. The icosahedral equation can be developed in terms of $\tau$, and $\tau$ is therefore the icosahedral irrationality.*

18. Now project $Q_{5}$ by planes on $L$, upon $\pi$. Since a plane in $S_{4}$ in general meets another plane in $S_{4}$ in only one point, involutory pairs on $Q_{5}$ project into single points. The locus of these points on $\pi$ is the conic whose parametric expression (parameter $z$ ) is furnished by $y_{i}$ of (29). The point equation of this doubly-covered conic is

$$
2 \tau_{1}^{2} \tau_{2}^{2} y_{0}^{2}-\tau_{1} \tau_{2}^{3} y_{1}^{2}+\tau_{1}^{3} \tau_{2} y_{2}^{2}+\tau_{1}^{4} y_{0} y_{1}-\tau_{1}^{2} \tau_{2}^{2} y_{1} y_{2}+\tau_{2}^{4} y_{0} y_{2}=0
$$

The line $L$ meets $Q_{5}$ in a singular point of $Q_{5}$, whose elliptic parameter is $u=0$, and whose parameter in (29) is $z=\tau$. The locus of the projection of this point on $\pi$ is the rational sextic

$$
\begin{aligned}
& y_{0}=-5 z_{1}^{3} z_{2}^{3}, \\
& y_{1}=z_{1}^{6}+3 z_{1} z_{2}^{5}, \\
& y_{2}=3 z_{1}^{5} z_{2}-z_{2}^{6} .
\end{aligned}
$$

The conic (31) is the osculant conic of (32) at the point $z=\tau$. With every $Q_{5}$ in the family is associated a point of the sextic (32). Hence

The family of elliptic quintics in $S_{4}$, which admit a given $G_{50}$ of collineations, is projected from the fixed line upon the fixed plane of one of the twenty-five involutory collineations of $G_{50}$ into a family of osculant conics of a rational

* See B. also. 
sextic in that plane. Through every point of the plane pass four conics of the system.

19. A consideration of the projection of $Q_{5}$ leads to a determination of the order $r$ of the surface $\psi$ which is the locus of the family of $Q_{5}$ 's. Since $L$ is on $\psi$, an $S_{3}$ on $L$ cuts $\psi$ in $L$ and some curve $C$ of order $r-1$, which meets $L$ in $s$ points. In a given $S_{3}$, an $S_{2}$ on $L$ cuts $C$ in $s$ points on $L$ and $r-1-s$ further points. But it has been noted that through every point of $\pi$ pass four conics and that to every point of a conic corresponds two points of a particular $Q_{5}$. Hence to every point of the plane corresponds eight points of the surface $\psi$. Therefore

$$
r-1-s=8 .
$$

Now the $S_{3}$ on $L$, cutting $\psi$ in $L$ and $C$ contains two directions on the surface $\psi$ at every point of intersection of $L$ and $C$, and therefore contains the tangent $S_{2}$ ar such a point. But the section of $S_{3}$ by $\pi$ is a line and the projection of points of contact of tangent planes is the sextic (32). Since a line cuts a sextic in six points, $s=6$.

$$
\therefore \quad r=15 \text {. }
$$

20. In the preceding paragraph one line, $L$, on $\psi$ has been mentioned. This however is but one of twenty-five such lines which are the fixed lines of the involutory collineations in $G_{50}$. These lines lie by fives on $S_{3}$ 's. Five $S_{3}$ 's containing such an arrangement of these lines form one of the six fundamental pentahedra.

Another set of lines on $\psi$ are those composing the degenerate $Q_{5}$ 's obtained by letting $\tau$ equal the roots of

$$
\alpha_{\tau}^{12} \equiv \tau_{1} \tau_{2}\left(\tau_{1}^{10}+11 \tau_{1} \tau_{2}-\tau_{2}^{10}\right)=0 .
$$

Each degenerate curve is a skew pentagon. The twelve pentagons can be arranged in six pairs, each pair forming edges of a fundamental pentahedron. The pairs are given by

and

$$
\tau_{1} \tau_{2}=0
$$

$$
\begin{aligned}
{\left[\tau_{1}-\epsilon^{\nu}\left(\epsilon+\epsilon^{4}\right) \tau_{2}\right]\left[\tau_{1}-\epsilon^{\nu}\left(\epsilon^{2}+\epsilon^{3}\right) \tau_{2}\right] } & =0 \\
(\nu & \left.=0, \cdots 4 ; \epsilon=e^{(2 \pi i) / 5}\right) .
\end{aligned}
$$

21. In a study of the configurations in the plane $\pi$, the transformations arising through the modular substitutions $\boldsymbol{S}$ and $\boldsymbol{T}$ are valuable. Bianchi has given the two transformations also called $S$ and $T$ in terms of $x$. They with $C_{1}, C_{2}, C_{3}$ generate $G_{50} \cdot 60$ under which the members of the family of $Q_{5}$ 's in $S_{4}$ are at the most permuted. Under $G_{50} \cdot 60, G_{50}$ is an invariant subgroup. The factor group is a $G_{60}$ isomorphic with the modular substitu 
tions. The subgroup of $G_{50.060}$ leaving $C_{1}$ unaltered is a $G_{2} \cdot 60$. It has an invariant $G_{2},\left\{1, C_{1}\right\}$, whose factor group is isomorphic with the $G_{60}$ already mentioned. This may be represented either by the transformations on $z_{1}: z_{2}$ or those on $y_{0}: y_{1}: y_{2}$. The substitutions in $\pi$ which generate such a $G_{60}$ are obtained by setting $z_{1}=z_{2}=0$ in $T$ and $\left(C_{1} S^{2}\right)^{3} \equiv S_{1}^{\prime}$. The explicit expressions are

$$
\begin{array}{|l|l|l|}
\multicolumn{1}{c|}{\boldsymbol{S}_{1}^{\prime}} & \multicolumn{1}{c|}{\boldsymbol{T}^{\prime}} \\
\hline y_{0}^{\prime} & =y_{0} & =y_{0}+y_{1}+y_{2} \\
y_{1}^{\prime} & =\boldsymbol{\epsilon}^{2} y_{1} & =2 y_{0}+\left(\boldsymbol{\epsilon}+\boldsymbol{\epsilon}^{4}\right) y_{1}+\left(\boldsymbol{\epsilon}^{2}+\boldsymbol{\epsilon}^{3}\right) y_{2} \\
y_{2}^{\prime} & =\boldsymbol{\epsilon}^{3} y_{2} & =2 y_{0}+\left(\boldsymbol{\epsilon}^{2}+\boldsymbol{\epsilon}^{3}\right) y_{1}+\left(\boldsymbol{\epsilon}^{4}+\boldsymbol{\epsilon}\right) y_{2} \\
z_{1}^{\prime} & =-\boldsymbol{\epsilon}^{2} z_{1} & =\left(\boldsymbol{\epsilon}-\boldsymbol{\epsilon}^{4}\right) z_{1}+\left(\boldsymbol{\epsilon}^{3}-\boldsymbol{\epsilon}^{2}\right) z_{2} \\
z_{2}^{\prime} & =-\boldsymbol{\epsilon}^{3} z_{2} & =\left(\boldsymbol{\epsilon}^{3}-\boldsymbol{\epsilon}^{2}\right) z_{1}+\left(\boldsymbol{\epsilon}^{4}-\boldsymbol{\epsilon}\right) z_{2}{ }^{*}
\end{array}
$$

Let us now consider the degenerate $Q_{5}$ 's and their projections in $\pi$. To particularize let $\tau_{1}=0$ or $\tau_{2}=0$. Either condition applied to $\phi_{i}$ gives the five $S_{3}$ 's of $P_{\infty}$, the reference pentahedron in the $x$ coördinate system. These $S_{3}$ 's combined three at a time give the ten linest which in terms of $y_{i}$ and $z_{j}$ are

\begin{tabular}{rrrrrr} 
& $q_{1}^{\prime}$ & $q_{2}^{\prime}$ & $q_{3}^{\prime}$ & $q_{4}^{\prime}$ & $q_{5}^{\prime}$ \\
$Q_{5}^{\prime}$ & $y_{0}=0$ & $y_{0}=0$ & $y_{1}=0$ & $y_{1}=0$ & $y_{0}=0$ \\
& $y_{2}=0$ & $y_{1}+z_{1}=0$ & $y_{2}+z_{2}=0$ & $y_{2}-z_{2}=0$ & $y_{2}-z_{2}=0$ \\
& $z_{2}=0$ & $y_{2}+z_{2}=0$ & $y_{1}-z_{1}=0$ & $z_{1}=0$ & $y_{1}-z_{1}=0$ \\
\cline { 2 - 5 } & $q_{1}^{\prime \prime}$ & $q_{2}^{\prime \prime}$ & $q_{3}^{\prime \prime}$ & $q_{4}^{\prime \prime}$ & $q_{5}^{\prime \prime}$ \\
$Q_{5}^{\prime \prime} \quad$ & $y_{0}=0$ & $y_{0}=0$ & $y_{1}+z_{1}=0$ & $y_{2}=0$ & $y_{0}=0$ \\
& $y_{1}=0$ & $y_{1}+z_{1}=0$ & $y_{2}=0$ & $z_{2}=0$ & $y_{2}+z_{2}=0$ \\
& $z_{1}=0$ & $y_{2}-z_{2}=0$ & $z_{2}=0$ & $y_{1}-z_{1}=0$ & $y_{1}-z_{1}=0$ \\
\hline
\end{tabular}

The double points of the degenerate curves are the vertices of $P_{\infty}$. They project into the vertices of a triangle, $\Delta_{\infty}$, the reference triangle in the fixed

* It is noteworthy that the transformations $T_{1}^{\prime}$ and $S_{1}^{\prime}$ on the ternary variables $y_{i}$ are contragredient to the corresponding transformations on three quadratics in the binary domain, e. g., if $A_{0}=-z_{1} z_{2}, A_{1}=z_{2}^{2}, A_{2}=-z_{1}^{2}$ the result of operating with $T^{\prime}$ is

$$
\begin{gathered}
A_{0}^{\prime}=A_{0}+A_{1}+A_{2}, \quad A_{1}^{\prime}=2 A_{0}+\left(\epsilon^{2}+\epsilon^{3}\right) A_{1}+\left(\epsilon+\epsilon^{4}\right) A_{2}, \\
A_{2}^{\prime}=2 A_{0}+\left(\epsilon+\epsilon^{4}\right) A_{1}+\left(\epsilon^{2}+\epsilon^{3}\right) A_{2} .
\end{gathered}
$$

The result of operating with $S_{1}^{\prime}$ is

$$
A_{0}^{\prime}=A_{0}, \quad A_{1}^{\prime}=\epsilon A_{1}, \quad A_{2}^{\prime}=\epsilon^{4} A_{2} .
$$

See Klein Vorlesungen über das Ikosaeder, pp. $211 \mathrm{ff}$.

$\dagger$ The lines are ordered as is indicated by the subscripts. The arrangement given is the unique one in which the ten lines form two skew pentagons so that all the lines are included, none are repeated. It was determined by a consideration of corresponding points and lines under $C_{1}^{\prime}$. 
plane $\pi$ of $C_{1}^{\prime}$. The vertices $e_{1}$ and $e_{2}$ of $\Delta_{\infty}$ are doubly covered, $e_{0}$ singly covered. The lines $q_{1}^{\prime}$ and $q_{1}^{\prime \prime}$ are unaltered by $C_{1}^{\prime}$, while the remaining lines of each degenerate curve are interchanged by pairs. The lines $q_{1}^{\prime}$ and $q_{1}^{\prime \prime}$ cut $\pi$ in the points $e_{1}$ and $e_{2}$ which are therefore the projections of $q_{1}^{\prime}$ and $q_{1}^{\prime \prime}$ respectively. The table shows the entire projection of $Q_{5}^{\prime}$ and $Q_{3}^{\prime \prime}$.

$$
\begin{array}{cccccccc}
q_{1}^{\prime} & \text { projects into } & e_{1}, & \multicolumn{3}{c}{q_{1}^{\prime \prime}} & \text { projects into } & e_{2}, \\
q_{2}^{\prime} & \text { “ } & \text { “ } & \epsilon_{0}, & q_{2}^{\prime \prime} & \text { “ } & \text { “ } & \epsilon_{0}, \\
q_{3}^{\prime} & \text { “ } & \text { “ } & \epsilon_{1}, & q_{3}^{\prime \prime} & \text { “ } & \text { “ } & \epsilon_{2}, \\
q_{4}^{\prime} & \text { “ } & \text { “ } & \epsilon_{1}, & q_{4}^{\prime \prime} & \text { “ } & \text { “ } & \epsilon_{2}, \\
q_{5}^{\prime} & \text { “ } & \text { “ } & \epsilon_{0}, & q_{5}^{\prime \prime} & \text { “ } & \text { “ } & \epsilon_{0} .
\end{array}
$$

Now the triangle $\Delta_{\infty}$ is also obtained by taking the intersections of the $S_{3}$ 's of $P_{\infty}$ with $\pi$. Since $P_{i}$ can be obtained from $P_{\infty}$ by operating with $T S^{i}$, the remaining five triangles, $\Delta_{i}$, in $\pi$ can be found by operating on $\Delta_{\infty}$ with the substitutions in the plane which correspond to $T S^{i}$ of $S_{4}$.

It has been seen that $z=\tau$ gives the tangent plane at the point where the fixed line $L$ of $C_{1}^{\prime}$ cuts $Q_{5}$ (vide $\S 18$ ). For $Q_{5}^{\prime}$ and $Q_{5}^{\prime \prime}, L$ cuts $q_{1}^{\prime}$ and $q_{1}^{\prime \prime}$. But $\tau_{2}=z_{2}=0$ gives in $\pi$ the point $e_{1}$ and $\tau_{1}=z_{1}=0$ gives in $\pi$ the point $e_{2}$ (vide (29)). Hence the modulus attached to $Q_{5}^{\prime}$ is $\tau_{2}=0$, to $Q_{5}^{\prime \prime}$ is $\tau_{1}=0$.

In the triangle $\Delta_{\infty}$ the side $\epsilon_{0}$ is isolated. This isolated side is a double flex tangent of the sextic (32). The parameters of the two flexes are $z_{1}=0$, $z_{2}=0$. The parameters of all the flexes of (32) are given by the icosahedral form $\alpha_{z}^{12}$. The remaining double flex tangents are derived from $y_{0}=0$ by operating with $\boldsymbol{T}^{\prime} \boldsymbol{S}^{\prime \nu}(\nu=0, \cdots, 4)$. The result is

$$
y_{0}+\epsilon^{2 \nu} y_{1}+\epsilon^{3 \nu} y_{2}=0 .
$$

The six double flex tangents are isomorphic with the six lines of Klein's icosahedral configuration in the plane* (vide Klein, Vorlesungen über das Ikosaeder, pp. $211 \mathrm{ff})$. The invariant conic of $G_{60}$ in the plane is the conic on the flexes

$$
y_{0}^{2}+y_{1} y_{2}=0 .
$$

It cuts out on the sextic the icosahedral points.

In the plane $\pi$ lie three double points of the involution $C_{1}^{\prime}$. They can be obtained by setting $z_{1}=z_{2}=0$ in $\phi_{0}^{\prime}, \phi_{1}^{\prime}, \phi_{2}^{\prime}$. If from the forms $\phi_{i}^{\prime}$ thus modified, $\tau_{1}^{2}, \tau_{1} \tau_{2}, \tau_{2}^{2}$ are eliminated, a sextic is obtained as the locus of the three fixed points of $C_{1}^{\prime}$, lying in $\pi$, for the whole family of $Q_{5}$ 's. Its equation is

* The rest of the configuration is not particularly interesting in connection with $Q_{5}$ so it is not given. A more complete discussion of the sextic (32) is given by R. M. Winger, Selfprojective rational sextics, American Journal of Mathematics, vol. 38, January, 1916. 


$$
y_{0}\left(y_{1}^{5}+y_{2}^{5}\right)+2 y_{0}^{2} y_{1}^{2} y_{2}^{2}-8 y_{0}^{4} y_{1} y_{2}-y_{1}^{3} y_{2}^{3}=0 \text {. }
$$

In $\S 18$ the point $z=\tau$ on the doubly-covered conic (31) was accounted for. Its locus was (32), the locus of the fixed point of $C_{1}^{\prime}$ found on $L$. The fixed points of $C_{1}^{\prime}$ found on $\pi$ are given by $\alpha_{\tau}^{9} \alpha_{z}^{3}=0$, and their locus has now been found to be (33).

22. Return for a moment to a consideration of the projection of $Q_{5}$ by a pencil of $S_{3}$ 's on $\pi$,

$$
t_{2} z_{1}=t_{1} z_{2}
$$

to determine what happens when $t$ is a root of $\alpha_{t}^{12}=0$.

The fixed planes of the involutory collineations of $G_{50}$ in $S_{4}$ lie by fives on the five vertices of $P_{\infty}$. There are as many such arrangements as there are pentahedra. Hence there are thirty points on each of which is a fixed plane. In every plane there are six points. The twenty-four points other than the six in the plane $z_{1}=0, z_{2}=0$, lie by pairs on $S_{3}$ 's in the pencil (30) where $t$ assumes the values of the roots of $\alpha_{t}^{12}=0$. 